\title{
ElectroWeak Symmetry Breaking in Tevatron Run II
}

\author{
Giorgio Chiarelli for the CDF and D0 Collaborations * \\ INFN,Sez.di Pisa, S.Piero a Grado(Pisa), Italy, Via Livornese 1291, 56010 \\ E-mail: 'giorgio.chiareíli@pi.infn. iti
}

Abstract: The Tevatron Run II will provide CDF and D0 with a large dataset of $p \bar{p}$ interactions collected at $\sqrt{s}=2 \mathrm{TeV}$. We discuss the opportunity for the two experiments to improve the understanding of electroweak and top physics in the first years of data taking (Run IIa, $2 \mathrm{fb}^{-1}$ ) in view of the upgrades of the detectors. We also discuss the prospectives for a Higgs discovery at the Tevatron in view of the Run IIb data taking period which will deliver an additional of about $13 \mathrm{fb}^{-1}$ to each experiment.

\section{Run II of the Tevatron Collider}

The successful Run I of the Tevatron in 1992-1996 led to the upgrade of the accelerator complex. The new run, started in March 2001, is known as Run II and is expected to collect $2 \mathrm{fb}^{-1}$ in a first phase (Run IIa) and, after another set of improvements to machine and detectors, gather additional $13 \mathrm{fb}^{-1}$ or so in what is known as Run IIb. The net results of the upgrades are an increase in energy (from $\sqrt{s}=1.8$ to $2 \mathrm{TeV}$ ) and in instantaneous luminosity (from a typical $10^{31}$ in Run I up to $5 \times 10^{32} \mathrm{~cm}^{-2} \mathrm{~s}^{-1}$ in Run IIb). The average number of interactions per crossing is kept low by decreasing the inter bunch distance from $3.5 \mu s$ of Run I down to $396 \mathrm{~ns}$ and which will eventually become $132 \mathrm{~ns}$.

In order to match the technical challenges posed by those changes, as well as to exploit the physics capabilities of the Tevatron, both CDF and D0 underwent a series of

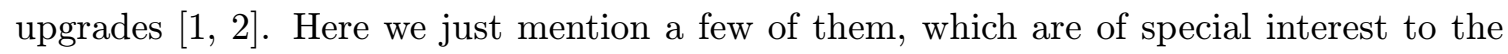
electroweak physics. CDF completely redesigned its front-end electronics and DAQ system to match the $132 \mathrm{~ns}$ interbunch. The trigger was also completely rebuilt, online tracking for hight $P_{t}$ tracks was moved to the first level trigger, while a new special set of processors, providing the experiment with a second level trigger on tracks displaced from the primary vertex (the Silicon Vertex Tracker), was constructed. Finally the whole tracking system was completely rebuilt. It is now made of a new silicon tracker ( 7 layers providing space points for $|\eta|<2$ at $2 \leq R \leq 28 \mathrm{~cm}$ ) and a new central drift chamber with a stronger 3D

\footnotetext{
${ }^{*}$ Speaker.
} 
reconstruction capability. Just to mention a few figure of merits, this system will allow tracking up to $|\eta|<2$ (therefore doubling the Run I coverage), and will increase the $\mathrm{b}$ tagging efficiency in top events to $65 \%$ per jet. The D0 upgrade was even more substantial as a solenoid, providing a $2 \mathrm{~T}$ magnetic field, was added. Futhermore the tracking system was rebuilt with a silicon vertex detector and a fiber tracker to fully exploit this new situation (figure iii). The D0 trigger, front end and DAQ systems were also redesigned to cope with the decreased bunch spacing. In the end the two experiments will have similar performances in terms of hermeticity and tracking capabilities.

To put things in perspective, compared to Run I the overall acceptance for W,Z events will be doubled and, for events containing a vector boson pair, it will be tripled.

\section{Gauge Boson Physics}

Run IIa with its $2 \mathrm{fb}^{-1}$ of data, will provide each experiment with over $3 \times 10^{6}$ W's (e and $\mu$ chan-

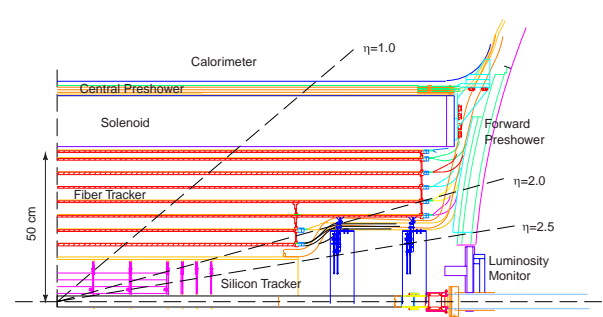

Figure 1: D0 upgrade nel), therefore allowing a precise determination of many EW parameters. One of the most important results will be a better determination of the W mass. In Run I CDF measured $M_{W}$ with an accuracy of $79 \mathrm{MeV}$, whereas D0's final accuracy was $84 \mathrm{MeV}$, bringing the total uncertainty for this measurement (Tevatron only) to $\simeq 60 \mathrm{MeV}$, with statistical and systematics on equal footing. However, since most of the systematic uncertainty depends upon the size of control samples, we will greatly benefit from the significantly larger data set. As an example we show (table $\left.\underline{1}_{i}^{\prime}\right)$ the contributions, essentially all scaling with lumi-

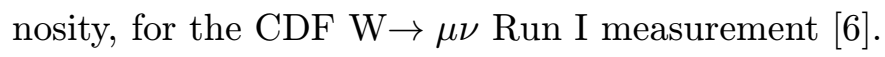

\begin{tabular}{|l|c|}
\hline Source & $\begin{array}{c}\text { uncert. } \\
\left(\mathrm{MeV} / \mathrm{c}^{2}\right)\end{array}$ \\
\hline Fit statistics & 100 \\
\hline Momentum scale & 85 \\
\hline Recoil model & 35 \\
\hline Background & 25 \\
\hline Mom.resolution & 20 \\
\hline Selection bias & 18 \\
\hline
\end{tabular}

Table 1: Run I $M_{W}$ uncertainties

The biggest component (momentum scale) is obtained by fitting all the $l^{+} l^{-}$invariant mass spectrum, while $\mathrm{W}$ asymmetry is used to constrain PDF and the $\mathrm{Z} P_{t}$ spectrum is the input to the $\mathrm{W} P_{t}$ spectrum. With $2 \mathrm{fb}^{-1}$ we expect to reduce the overall uncertainty to 40 $\mathrm{MeV} / \mathrm{c}^{2}$, with $30 \mathrm{MeV} / \mathrm{c}^{2}$ as a possible target.

The study of $\mathrm{W}$ and $\mathrm{Z}$ couplings will benefit from the wider acceptances and the new features of the two detectors. The anomalous couplings in the $\mathrm{ZV}(\mathrm{V}=\mathrm{Z}, \gamma)$ vertex are parameterized in terms of $h_{30}$ and $h_{40}$. Any value different from zero of those couplings would signal new physics. In Run I D0 set a $95 \%$ C.L.limit for $h_{40}$ at about 0.3 and in Run IIa we expect to reach a limit to better than 0.03 by using several hundreds of reconstructed $\mathrm{Z} \gamma$ $\rightarrow e e(\mu \mu) \gamma$ events and a few $Z Z \rightarrow 4 l$ detected. In the $\mathrm{W}$ sector we expect to collect about 100 events $\mathrm{WW} \rightarrow l \nu l \nu$ and about 30 events WZ $\rightarrow l l l \nu$. Scaling from Run I analysis, we expect to set limit for anomalous couplings, parameterized as $\Delta k$ and $\lambda$ to $-0.12<\Delta k<0.18$ for $\lambda=0$ and $|\lambda|<0.09$ for $\Delta k=0$. One of the most important checks of the SM, still to be performed, is the detection of the zero amplitude in the $\mathrm{W} \gamma \rightarrow \gamma l \nu$ 
process. Due to specific SM cancellations, a dip appears in the angular distribution of the final states $(l, \gamma)$. CDF saw hints of this effect in Run I and we expect to collect about 3000 $W \gamma \rightarrow l \nu \gamma$ in Run IIa, therefore being able to isolate this process.

\section{Top Physics in Run II}

Right after the discovery of the top quark, both experiments swiftly moved on to measure its properties (cross section, mass and couplings). Some of those measurements were already systematic-limited at the end of Run I. We expect most of those systematics to scale with statistics as they are determined by the size of control samples and therefore studies of the sixth quark will greatly benefit from the increase of luminosity and in c.o.m. energy. Raising the energy from $\sqrt{s}=1.8$ to $2 \mathrm{TeV}$, the production cross section increases by $40 \%$, as the $g g$ scattering acquires a more prominent role.

Table $\underset{2}{\overline{2}}$ shows the number of top events expected in $2 \mathrm{fb}^{-1}$ per experiment. To put things in perspective, CDF collected 9 candidates dilepton events in Run I, and 76 were used in the $1+4 \mathrm{j}$ sample (the most important one to determine $\left.M_{t o p}\right)$ to reconstruct the top mass. The larger statistics will be obtained thanks to a wider acceptance and better tracking capability. The improved b-tagging effi-

\begin{tabular}{|l|r|}
\hline Channel & CDF $/$ D0 \\
\hline Dilepton $(e, \mu)$ & 155 \\
\hline Dilepton $(\tau)$ & 19 \\
\hline lepton $+\geq 3$ jets & 1520 \\
\hline lepton $+\geq 4$ jets & 1200 \\
\hline lepton $+\geq 3$ jets +1 btag & 990 \\
\hline lepton $+\geq 3$ jets +2 btag & 240 \\
\hline
\end{tabular}

Table 2: Yield of $t \bar{t}$ events in $2 \mathrm{fb}^{-1}$ ciency (for example at D0 $\epsilon_{b}$ will be about 60 $\%$ for $b$-jets with $P_{t}>40 \mathrm{GeV} / \mathrm{c}$ ) will greatly improve, besides the determination of $M_{t o p}$, the measurement of the cross section and the study of the $W t b$ vertex.

The measurement of $M_{t o p}$ is dominated by systematics, with the biggest one being the jet energy scale. The best way to tackle this problem is to identify a signal on which calibrate the energy scale. In Run I CDF was able to reconstruct a $W \rightarrow j j$ peak in its tiny $t \bar{t}$ double $b$-tagged sample and to detect the $Z \rightarrow b \bar{b}$ signal in its dijet sample. Both experiments plan to do even better in Run II by using a dedicated trigger to select $Z \rightarrow b \bar{b}$ events. In this way the two vector bosons will be used to set the jet energy scale and to check $b$-specific corrections. Given that, we expect the uncertainty to

\begin{tabular}{|l|r|}
\hline Source & $\mathbf{G e V} / \mathbf{c}^{2}$ \\
\hline Jet En. scale & $4.4(2.2)$ \\
\hline ISR and FSR & $1.8(1.0)$ \\
\hline background & $1.3(0.5)$ \\
\hline b-tag bias & 0.4 \\
\hline PDF & 0.3 \\
\hline Total & $4.9(2.5)$ \\
\hline
\end{tabular}

Table 3: Run I systematics of CDF $M_{\text {top }}$ measurement.In parenthesis expectations in $2 \mathrm{fb}^{-1}$. be reduced by a factor 2 (see table $13 \overline{-1}$ for CDF estimates of expected systematics in Run IIa; D0's number are similar). Together with the precise determination of $M_{W}$ this will set stringent limit on the Higgs mass (see figure 2 2i).

Another topic in top physics which will be explored in Run II is the precision determination of the top cross section, which is currently $\mathrm{a} \simeq 25 \%$ measurement and which will be determined to better than $10 \%$ at the end of Run IIa. The comparison between collider data and Monte Carlo will improve with the availability of larger samples of $b$ tagged 
events, the wider acceptance and unprecedented MC samples both in level of detail and size. Studies of the $W t b$ vertex in Run I were performed at CDF. Both top helicity and

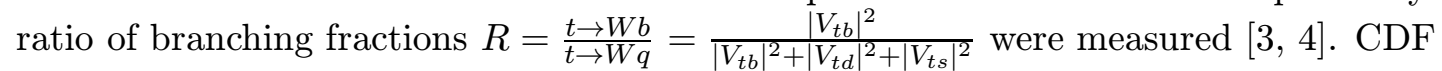
measurement of $R$ to $26 \%$ allowed an indirect determination of the CKM matrix element $\left|V_{t b}\right|$ to $\simeq 15 \%$. In Run II we expect to reduce the uncertainty on $R$ to $6 \%$ with a corresponding uncertainty on $\left|V_{t b}\right|$ to about $3 \%$. A direct determination of $\left|V_{t b}\right|(15 \%$ accuracy) can be obtained by identification of the single top production process, which however stands as an elusive process. The understading of forward tracking and tagging will play a decisive role in this search.

\section{Higgs Searches in Run II}

Over the last two years a lot of effort was dedicated to better understand the chances to detect a SM Higgs particle at the Tevatron. Emphasis was set on the low mass $\left(<130 \mathrm{GeV} / \mathrm{c}^{2}\right)$ region, where the Higgs decays almost completely into $b \bar{b}$ pairs. Although (figure Bij.) $_{j}$ the dominant production process is the direct production through $g g$ fusion, the large QCD background renders this channel unfeasible.

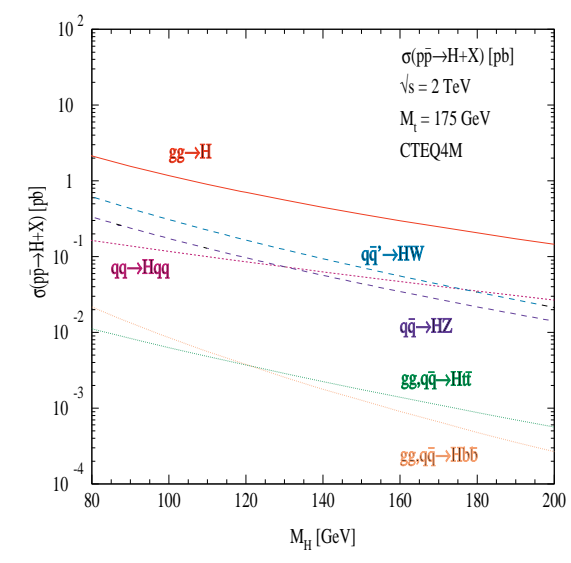

Figure 3: SM Higgs production

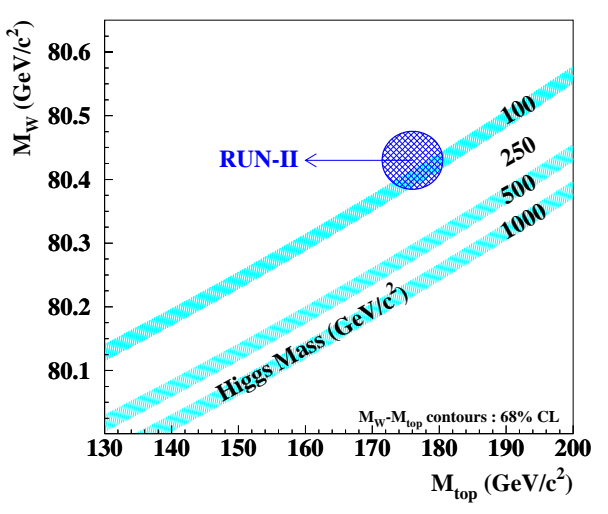

Figure 2: SM Higgs as a function of $M_{W}$ and $M_{\text {top }}$. Run IIa $\left(2 \mathrm{fb}^{-1}\right)$, one experiment only.

The associate production $V H(V=W, Z)$, where a leptonic decay of the $V$ would provide a clean trigger and bring to a favourable $\mathrm{S} / \mathrm{B}$ ratio, is more promising. A detailed study, based on a mix of Monte Carlo paremeterization, Run I data analysis and comparison with simulation of the CDF and D0 detector, was performed in the last three years. It is not possible here to describe all the studies performed [ini], the summary is provided in tables 4 and 5 where the event yield in $15 \mathrm{fb}^{-1}$ is shown for the $\mathrm{WH}$ and $\mathrm{ZH}$ case $(b \bar{b}$ background not shown but included in the significance). It is important to keep in mind that all, but the single top, background processes were already seen or measured in Run I. Therefore the calculation of the signal includes all known effects. The studies of the "high mass" region focused on the $g g \rightarrow H \rightarrow W^{*} W^{*} \rightarrow$ $l^{+} l^{-} \nu \nu$ channel where the price paid for the low cross section is offset by the very small background. An additional step was to add the associate production of $\mathrm{H}$ and $\mathrm{W}$ or $\mathrm{Z}$ : $p \bar{p} \rightarrow W(Z) H \rightarrow l^{ \pm} l^{ \pm} j j X$. By vetoing on $b$-tagged jets (produced in $t \bar{t}$ events), the backgrounds are reduced to a manageable level. The final outcome of these studies is shown in figure 


\begin{tabular}{|l|r|r|r|}
\hline$M_{H}\left(\mathbf{G e V} / \mathbf{c}^{2}\right)$ & $\mathbf{1 1 0}$ & $\mathbf{1 2 0}$ & $\mathbf{1 3 0}$ \\
\hline Signal & 75 & 60 & 45 \\
\hline$W b \bar{b}$ & 435 & 375 & 285 \\
\hline WZ & 90 & 60 & 30 \\
\hline$t \bar{t}$ & 225 & 300 & 330 \\
\hline single top & 105 & 135 & 135 \\
\hline S/ $\sqrt{B}$ & 2.6 & 2.0 & 1.6
\end{tabular}

Table 4: Higgs yield in $W H$ channel

\begin{tabular}{|l|r|r|r|}
\hline$M_{H}\left(\mathbf{G e V} / \mathbf{c}^{2}\right)$ & $\mathbf{1 1 0}$ & $\mathbf{1 2 0}$ & $\mathbf{1 3 0}$ \\
\hline Signal & 69 & 46 & 31 \\
\hline$Z b \bar{b}$ & 84 & 69 & 52. \\
\hline$W b \bar{b}$ & 100 & 81 & 63 \\
\hline $\mathrm{ZZ}$ & 43.5 & 3 & 0.0 \\
\hline$t \bar{t}$ & 70.5 & 64.5 & 52.5 \\
\hline single top & 79.5 & 70.5 & 57. \\
\hline S/ $\sqrt{B}$ & 2.4 & 2.0 & 1.5
\end{tabular}

Table 5: Higgs yield in $Z H$ channel

as a function of the Higgs mass and of the integrated luminosity. The two experiments have been combined. There is a window of opportunity in the low and intermediate Higgs mass region, if the Tevatron can provide an integrated luminosity in excess of 10-15 $\mathrm{fb}^{-1}$ before the start of the LHC. In order to do so the Beams Division of Fermilab initiated an intensive $R \& D$ program to demonstrate the feasibility of delivering about $4 \mathrm{fb}^{-1}$ per year. At the same time both CDF and D0

started an intensive period of studies to understand which parts of their detectors need to be replaced to survive luminosities more than 7 times larger than originally planned.

\section{Conclusion}

In the next years of Run II we expect to gather more than $2 \mathrm{fb}^{-1}$ per experiment. Thanks to the very large statistics precise determinations of the masses of the top quark and of the $W$

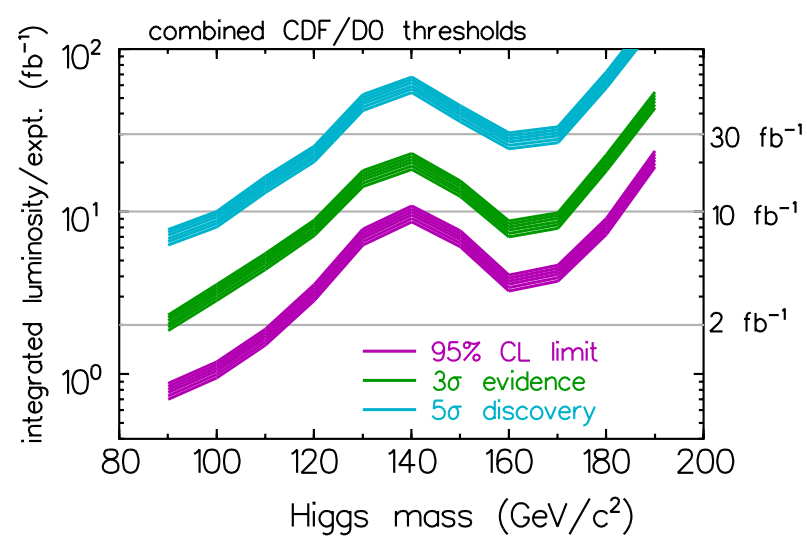

Figure 4: SM Higgs expectations at the Tevatron vector boson will be possible. This will allow to set stringent limit on the Higgs mass. Furthermore, as the Tevatron collider will be upgraded to deliver $\simeq 4 \mathrm{fb}^{-1}$ per year, there is a chance to isolate the Higgs particle if its mass is low enough.

\section{References}

[1] F.Abe et al., CDFII Technical Design Report, FERMILAB-96-390-E.

[2] M.Abolins et al., The Do Upgrade, FERMILAB-96-357-E.

[3] T.Affolder et al., Physs. Rev. Lett. 86 2001$) 3233$.

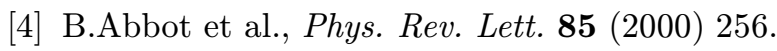

[5] M.Carena et al. Report of the Run II Higgs Working Group, hep-ph/0010338

[6] T.Affolder et al. 'P $\bar{h} \overline{y s}$. Rev. $\mathbf{D} \mathbf{6} \overline{4}-(\overline{2} \overline{0} \overline{1}) \overline{0} \overline{2} \overline{0} \overline{0} \overline{1}$ 